\title{
A protein complex of SCRIB, NOS1AP and VANGL1 regulates cell polarity and migration, and is associated with breast cancer progression
}

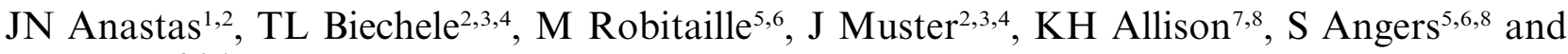 \\ RT Moon ${ }^{2,3,4}$ \\ ${ }^{1}$ Molecular and Cellular Biology Graduate Program, University of Washington School of Medicine, Seattle, WA, USA; ${ }^{2}$ Institute \\ for Stem Cell and Regenerative Medicine, University of Washington School of Medicine, Seattle, WA, USA; ${ }^{3}$ Department of \\ Pharmacology, University of Washington School of Medicine, Seattle, WA, USA; ${ }^{4}$ Howard Hughes Medical Institute, University of \\ Washington School of Medicine, Seattle, WA, USA; ${ }^{5}$ Department of Pharmaceutical Sciences, University of Toronto, Toronto, \\ Ontario, Canada; ${ }^{6}$ Department of Biochemistry, University of Toronto, Toronto, Ontario, Canada and ${ }^{7}$ Department of Pathology, \\ University of Washington School of Medicine, Seattle, WA, USA
}

By analyzing public data sets of gene expression in human breast cancers we observed that increased levels of transcripts encoding the planar cell polarity (PCP) proteins SCRIB and VANGL1 correlate with increased risk of patient relapse. Experimentally, we found that reducing expression of SCRIB by short-hairpin RNAs (shRNAs) reduces the growth of human breast cancer cells in xenograft assays. To investigate SCRIB-associated proteins that might participate in the responses of breast cancer cells to altered levels of SCRIB, we used mass spectrometry and confocal microscopy. These studies reveal that SCRIB is present in at least two unique protein complexes: (1) a complex of SCRIB, ARHGEF, GIT and PAK (p21-activated kinase), and (2) a complex of SCRIB, NOS1AP and VANGL. Focusing on NOS1AP, we observed that NOS1AP colocalizes with both SCRIB and VANGL1 along cellular protrusions in metastatic breast cancer cells, but does not colocalize with either SCRIB or VANGL1 at cell junctions in normal breast cells. We investigated the effects of shRNA-mediated knockdown of NOS1AP and SCRIB in vitro, and found that reducing NOS1AP and SCRIB slows breast cancer cell migration and prevents the establishment of leading-trailing polarity. We also find that reduction of NOS1AP enhances anchorage-independent growth. Collectively these data point to the relevance of NOS1AP and SCRIB protein complexes in breast cancer.

Oncogene (2012) 31, 3696-3708; doi:10.1038/onc.2011.528; published online 19 December 2011

Keywords: SCRIB; NOSIAP; VANGL1; migration; PCP

Correspondence: Dr RT Moon, University of Washington School of Medicine, Campus Box 358056, 815 Mercer Street, Room S524, Seattle, WA 98109, USA.

E-mail: rtmoon@uw.edu

${ }^{8}$ These authors contributed equally to this work.

Received 22 December 2010; revised 5 August 2011; accepted 2 September 2011; published online 19 December 2011

\section{Introduction}

Maintenance of tissue polarity requires restriction of determinant proteins to specific subcellular regions. At a molecular level, localizing proteins to distinct regions involves the activity of scaffolds, including the PDZ domain-containing protein SCRIB (Scribble). In Drosophila, deletions in scrib result in defects in apicalbasal polarity (Bilder and Perrimon, 2000; Bilder et al., 2000, 2003), whereas simultaneous loss of scrib and van gogh (VANGL1/2, or Strabismus) disrupts the planar cell polarity (PCP) of wing bristles and ommatidial cells (Courbard et al., 2009). Comparable PCP defects arise owing to a loss of SCRIB orthologs during vertebrate development. Specifically, inactivating Scrib alleles lead to disorganized stereocilia in the mouse inner ear (Murdoch et al., 2001, 2003; Montcouquiol et al., 2003), and impaired convergent extension movements in zebrafish (Wada et al., 2005; Vervenne et al., 2008). The evolutionarily conserved role of SCRIB in establishing or maintaining the three-dimensional organization of tissues may stem from its function as a molecular bridge between different members of multiprotein complexes. In epithelial tissues, SCRIB is recruited to sites of cell contact where it associates with junctional proteins (Petit et al., 2005a, b; Vervenne et al., 2008). The disorganization of epithelial tissues owing to loss of function of SCRIB may in part result from the abnormal localization of junctional proteins such as E-cadherin and $\beta$-catenin (Bilder and Perrimon, 2000; Bilder et al., 2000; Métais et al., 2005; Qin et al., 2005; Ivanov et al., 2010). In addition to acting at sites of cell-cell contact, SCRIB also participates in protein complexes necessary to establish leading-trailing polarity during cell migration. In motile cells SCRIB associates with a complex of ARHGEF7 ( $\beta$-PIX), the closely related ARHGEF6 ( $\alpha$-PIX), PAK1-3 (p21-activated kinases) and GIT proteins (Audebert et al., 2004; Nola et al., 2008; Moreau et al., 2010). This complex acts as a crucial regulator of cell migration and invasiveness (Qin et al., 2005; Osmani et al., 2006; Dow et al., 2007; Nola et al., 2008; Phua et al., 2009). 
The ability of SCRIB to regulate both the threedimensional morphogenesis of tissues and the migration of cells in vitro (Qin et al., 2005; Osmani et al., 2006; Dow et al., 2007) and in vivo (Murdoch et al., 2001, 2003; Wada et al., 2005; Vervenne et al., 2008) makes it a prime candidate for participating in cancer progression. This possibility is supported by the results of the present study in which we first demonstrate that altered expression of SCRIB correlates with poor clinical outcomes in breast cancer. We then demonstrate that SCRIB associates with at least two distinct protein complexes, and that an NOS1AP, SCRIB and VANGL1 protein complex modulates the migration, polarity and growth of breast cancer cells.

\section{Results}

Expression of SCRIB is associated with adverse clinical outcomes in breast cancer

Although previous research indicates that SCRIB can act as a suppressor of tumor growth in a mouse model of breast cancer (Zhan et al., 2008), the clinical relevance of SCRIB in human cancers remains unclear. We therefore evaluated the relationship between SCRIB expression and breast cancer relapse using data from a publically available multiple microarray data set of 286 human breast tumors (GEO-GSE2034) (Wang et al., 2005). Using the Kaplan-Meier method for risk assessment we found that samples with the highest $S C R I B$ expression ( $>65$ th percentile, $n=100$ ) were associated with a statistically significant increased risk of relapse $(* P=0.0240)$ when compared with samples with the lowest $S C R I B$ expression ( $<35$ th percentile, $n=100$ ) (Figure 1a). We again used Kaplan-Meier analysis to investigate the relationship between $V A N G L 1$ expression and clinical outcomes, and found a similar association between high levels of $V A N G L 1$ and breast cancer relapse $(* * P=0.003)$ (Figure $1 b)$. Finally, we analyzed a second multiple tumor microarray data set (GEO-GSE3143) (Bild et al., 2006) to determine the relationship between $S C R I B$ expression and overall breast cancer survival, and found that samples with high $S C R I B$ expression ( $>65$ th percentile, $n=56$ ) were associated with decreased overall survival probability $(* P=0.0407)$ (Figure 1c). These data provided the impetus for the following initial inquiries into the effects of altered levels of SCRIB and associated proteins on breast cancer cells.

\section{Reduction of SCRIB inhibits the growth of human breast cancer cells in xenografts}

We first asked whether manipulating SCRIB protein levels alters the growth of human breast cancer cells in xenograft assays. To obtain breast cancer cell lines with reduced levels of SCRIB protein, we transduced MDAMB-231 cells with two independent short-hairpin RNAs (shRNAs) complementary to SCRIB (SCRIB sh3 and SCRIB sh4). Western blot of lysates of these cells established that levels of SCRIB were reduced when compared with cells transduced with a PLKO.1 control shRNA (Figure 2a). We then pooled equal numbers of SCRIB sh3 and SCRIB sh 4 cells, and injected them into the mammary fats pads of immunocompromised NOG mice. We found that reducing SCRIB protein levels significantly slows tumor growth estimated by volume from caliper-measured dimensions (Figure 2b) and by final tumor mass (Figure 2c) when compared with cells transduced with a PLKO.1 control shRNA. SCRIB shRNAs also inhibited xenograft tumor growth in athymic nude mice (Figure 2d), indicating that the effect of SCRIB shRNAs is not specific to the genetic background of the animal.

\section{Mass spectrometry reveals multiple SCRIB protein complexes}

As the consequences of altered SCRIB expression are likely to be mediated by its associated proteins, we characterized SCRIB protein complexes by mass spectrometry. Specifically, we immunoprecipitated both endogenous SCRIB and double epitope-tagged SCRIB from HEK293 T cells, and analyzed protein complexes by tandem mass spectrometry. First, the results confirm a recent report (Audebert et al., 2004) that both endogenous and ectopically expressed SCRIB co-purify with a complex of ARHGEF7, PAK and GIT proteins. Second, the mass spectrometry data indicate that several PCP pathway components co-purify with SCRIB, including the transmembrane proteins VANGL1, VANGL2 and CELSR2 (also known as Flamingo). Finally, we identified novel SCRIB-associated proteins, including, KCTD3 and TJP1 (Figure 3a, and Supplementary Tables S1 and S2).

We focused our further studies of SCRIB-associated proteins on NOS1AP owing to high confidence in the mass spectrometry data and because of the known roles for NOS1AP in signal transduction (Jaffrey et al., 1998; Fang et al., 2000; Jaffrey, 2002; Carrel et al., 2009; Richier et al., 2010). Analysis of NOS1AP complexes isolated from HEK293 T cells by mass spectrometry confirms that NOS1AP associates with SCRIB as previously published (Richier et al., 2010), and revealed a novel association between NOS1AP and VANGL1 (Figure 3a and Supplementary Table S4).

Next, we validated the protein interaction network discovered by mass spectrometry by expressing green fluorescent protein (GFP), or a GFP-NOS1AP fusion protein, in MDA-MB-231 breast cancer cells and immunoprecipitating protein complexes with GFP, SCRIB or IgG antibodies, followed by western blotting (Figure 3b). We observed that GFP-NOS1AP, but not a GFP control, co-purifies with SCRIB (Figure 3b, lanes 2 and 3 versus lanes 6 and 7) but not with control IgG immunoprecipitates (Figure 3b, lanes 4 and 8). We also confirmed that NOS1AP is present in a protein complex with VANGL1 in breast cancer cells by showing that endogenous VANGL1 co-immunoprecipitates with FgNOS1AP, but not with Fg-GFP or with Fg-ARHGEF7 (Figure 3d, compare lane-5 with lanes 4 and 6).

As a previous study reported that SCRIB acts as a molecular bridge that mediates the interaction between 

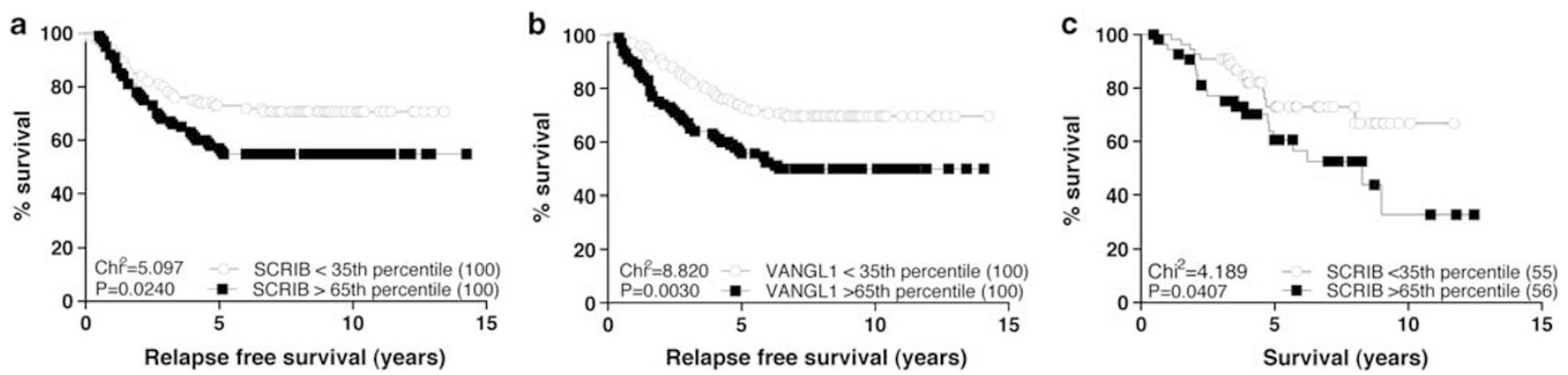

Figure 1 Expression of $S C R I B$ is associated with adverse clinical outcomes in breast cancer. (a) Analysis of a publically available breast cancer multiple tumor microarray data set (Wang et al., 2005) for association between SCRIB expression and risk of relapse. Samples were grouped as high $S C R I B$ expressing ( $>65$ th percentile, $n=100)$ and low SCRIB expressing $(<35$ th percentile, $n=100)$, and Kaplan-Meier curves were compared. $P=0.0240$ (log-rank test). (b) VANGL1 expression data analyzed as for SCRIB using the Wang et al. (2005) data set. $P=0.003$ (logrank test). (c) Kaplan-Meier survival analysis of a multiple tumor microarray data set of breast cancer samples (Bild et al., 2006) for association between $S C R I B$ expression and overall breast cancer patient survival. Samples were grouped as high $S C R I B$ expressing ( $>65$ th percentile, $n=56$ ) and low $S C R I B$ expressing ( $<35$ th percentile, $n=55)$, and curves were compared. $P=0.0407$ (log-rank test).

a
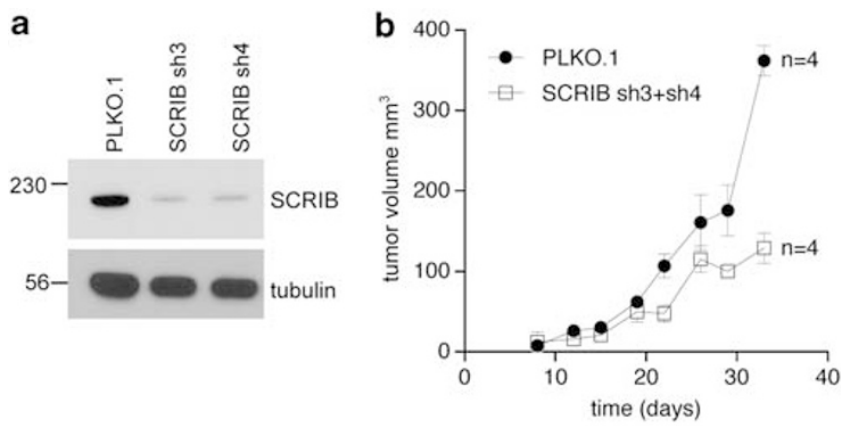

C
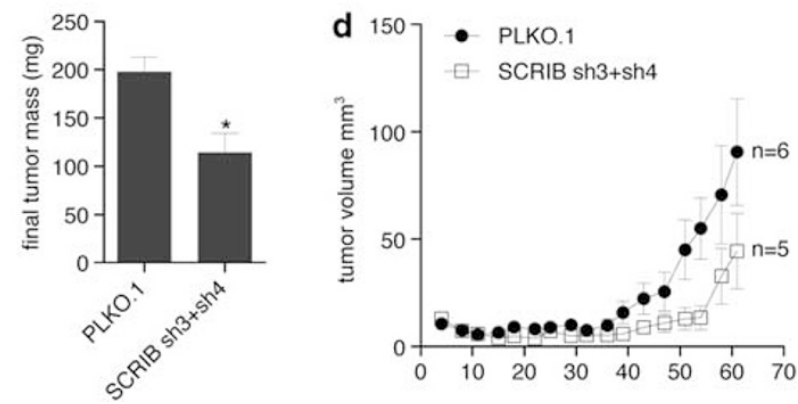

Figure 2 Reduction of SCRIB inhibits the growth of human breast cancer cells in xenografts. (a) Western blot of lysates from stable MDA-MB-231 cell lines infected with a PLKO.1 control shRNA, and two independent SCRIB shRNAs, analyzed for SCRIB expression (upper panel) or a $\beta$-tubulin loading control (bottom panel). (b) Tumor growth curves generated from mammary fat pad xenografts of shRNA-infected MDA-MB-231 cells in NOG immunocompromised mice $(n=4)$. (c) Final mass of orthotopic xenograft tumors, which were removed after killing the animals at the end of the growth experiment in panel $\mathbf{b}$. $* P<0.05$ (Student's $t$-test). (d) Tumor growth curves generated from mammary fat pad xenografts of shRNA-infected MDA-MB-231 cells in athymic nude immunocompromised mice $(n=6)$.

NOS1AP and ARHGEF7 (Richier et al., 2010), we tested whether NOS1AP also associates with ARHGEF7 in breast cancer cells. As NOS1AP did not co-immunoprecipitate with endogenous ARHGEF7 in MDA-MB-231 cells (Figure 3c), we asked instead whether NOS1AP and ARHGEF7 might independently associate with distinct SCRIB complexes. In order to test the hypothesis that SCRIB can interact with either ARHGEF7 or NOS1AP, but not both in the same complex, we performed a competition assay. For these experiments, we expressed different levels of Fg-NOS1AP or control Fg-GFP in HEK293 T cells, and found that, as the levels of Fg-NOS1AP, but not control FgGFP, increased, there was a reduction in the amount of endogenous ARHGEF7 that co-immunoprecipitated with SCRIB (Figure 3e, compare lanes 9-11 with lanes 12-14). These data suggest that NOS1AP competitively displaces ARHGEF7 from SCRIB complexes. Moreover, these co-immunoprecipitation experiments confirm our mass spectrometry results, and support the hypothesis that SCRIB associates independently with ARHGEF-PAK-GIT protein complexes and with NOS1AP-VANGL protein complexes.

\section{Subcellular localization of the SCRIB protein interaction network}

If SCRIB exists in multiple protein complexes as the above data suggest, then confocal microscopy of intact cells should reveal domains of overlap between SCRIB and potentially associated proteins. We characterized the subcellular localization of SCRIB and SCRIBassociated proteins in MDA-MB-231 breast cancer cells to investigate this hypothesis. Consistent with our protein interaction data, we found that NOS1AP localizes in a punctuate distribution throughout the cytoplasm and partially colocalizes with SCRIB at cellular protrusions (Figure 4a). VANGL similarly localizes to cell protrusions and partially colocalizes with both SCRIB and NOS1AP (Figures 4c and d). Also consistent with our protein interaction data, we observed colocalization of endogenous SCRIB and ARHGEF7 at lamellipodia in subsets of MDA-MB-231 cells (Figure $4 \mathrm{~b}$ and Supplementary Figure S2a). Consistent with the hypothesis that NOS1AP and ARHGEF7 interact with distinct pools of SCRIB, we observed minimal colocalization between ARHGEF7 and GFP-NOS1AP in breast cancer cells (Supplementary Figure S2b). 
a

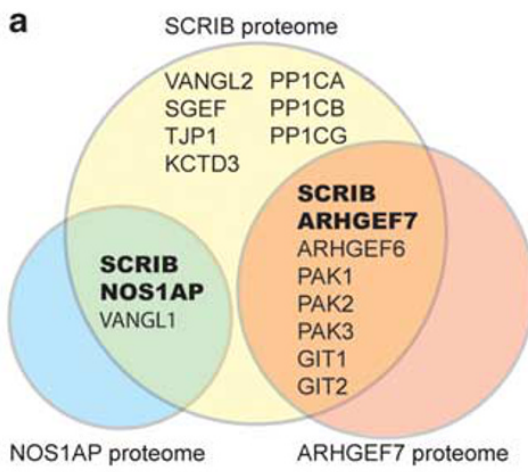

b

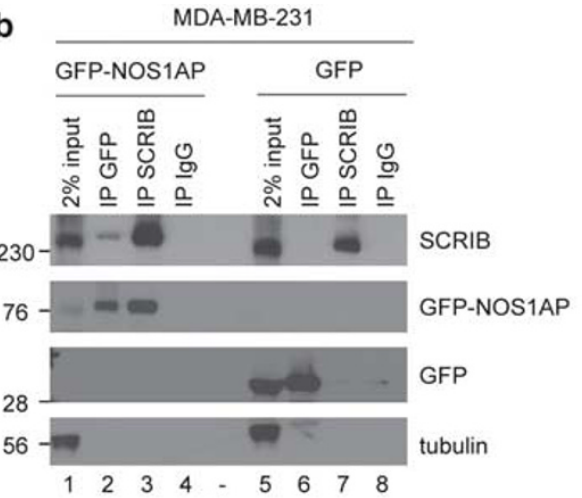

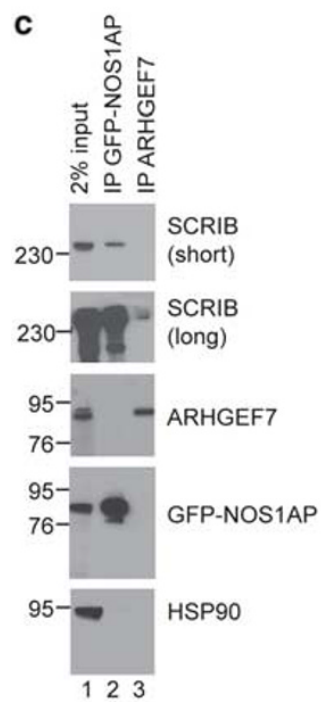

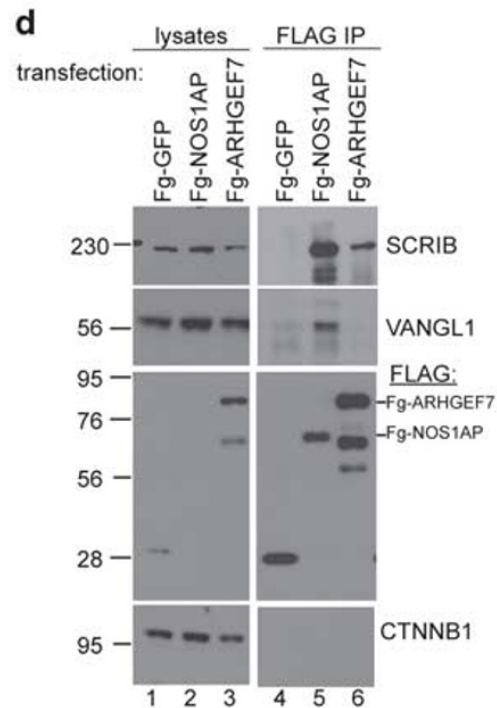

e

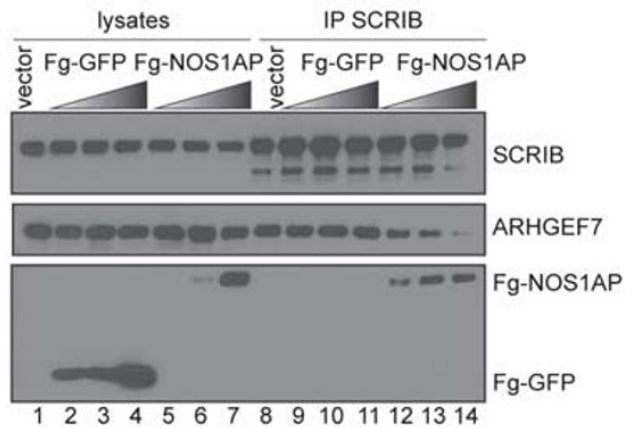

Figure 3 Mass spectrometry reveals multiple SCRIB protein complexes. (a) Venn diagram of protein interaction network generated from SCRIB, NOS1AP and ARHEGF7 mass spectrometry data. For peptide coverage information and detailed mass spectrometry results, see Supplementary Tables S1-S4. (b) Analysis of protein complexes isolated from MDA-MB-231 breast cancer cells expressing either GFP (lanes 5-8) or GFP-NOS1AP fusion protein (lanes 1-4). Protein complexes were isolated by immunoprecipitation using the following antibodies: GFP (lanes 2 and 6), SCRIB (lanes 3 and 7) or control IgG (lanes 4 and 8), and associated proteins were detected by western blotting for endogenous SCRIB (top panel), GFP and GFP-NOS1AP (middle panels), and $\beta$-tubulin (bottom panel). (c) Analysis of GFP-NOS1AP (lane-2) or endogenous ARHGEF7 protein complexes (lane-3) immunoprecipitated from MDA-MB231 cell lysates. Endogenous SCRIB (top two panels), ARHGEF7 (third panel from top), HSP90 (bottom panel) and ectopic GFPNOS1AP (fourth panel from top) were detected by western blotting. (d) Analysis of VANGL1 association using ectopically expressed FLAG (Fg) fusion proteins in breast cancer cells. Fg-GFP, Fg-NOS1AP or Fg-ARHGEF7 protein complexes were immunoprecipitated from MDA-MB-231 cell lysates, and the associated proteins were detected by western blotting for endogenous SCRIB, VANGL1 and CTNNB1, and for overexpressed FLAG. (e) Competitive binding experiment in HEK293 T cells transiently transfected with increasing amounts of Fg-GFP (bottom panel, lanes 2-4 and 9-11) or Fg-NOS1AP (bottom panel, lanes 5-7 and lanes 12-14). SCRIB was immunoprecipitated from these cells (lanes 8-14) and the amount of ARHGEF7 (middle panel) associated with SCRIB (upper panel) in the presence of increasing amounts of Fg-GFP (lanes 9-11) or Fg-NOS1AP (lanes 12-14) was analyzed by western blotting.

\section{NOS1AP is undetectable at cell junctions in mammary cells}

We next asked whether the association between SCRIB and NOS1AP was observed in normal mammary cells. Unlike invasive breast cancer cells, MCF10a cells form epithelial sheets with adherens junctions marked by CTNNB1 ( $\beta$-catenin) (Figure 5a). We found that SCRIB and VANGL1 localized to these sites of cell contact (Figures 5a and b), whereas NOS1AP remained mostly restricted to the cytosol (Figure $5 \mathrm{c}$ ). We also assessed the localization of SCRIB and NOS1AP in MCF-7 breast cancer cells, which retain their epithelial characteristics and found that SCRIB again colocalized to cell-cell junctions with CTNNB1, whereas a majority of the NOS1AP signal remained in the cytosol (Supplementary Figure S3).

We then used concanavalin-A beads to enrich for the plasma membrane and junctional complexes of mammary cells. We observed that a much greater proportion of SCRIB was bound to the concanavalin-A beads when we fractionated MCF10a cell lysates compared with when we fractionated MDA-MB-231 cell lysates (Figures $5 \mathrm{~d}$ and e). These data are consistent with the confocal microscopy data that show localization of SCRIB to the plasma membrane at cell-cell junctions in MCF10a cells (Figures 5a-c). Furthermore, we found 

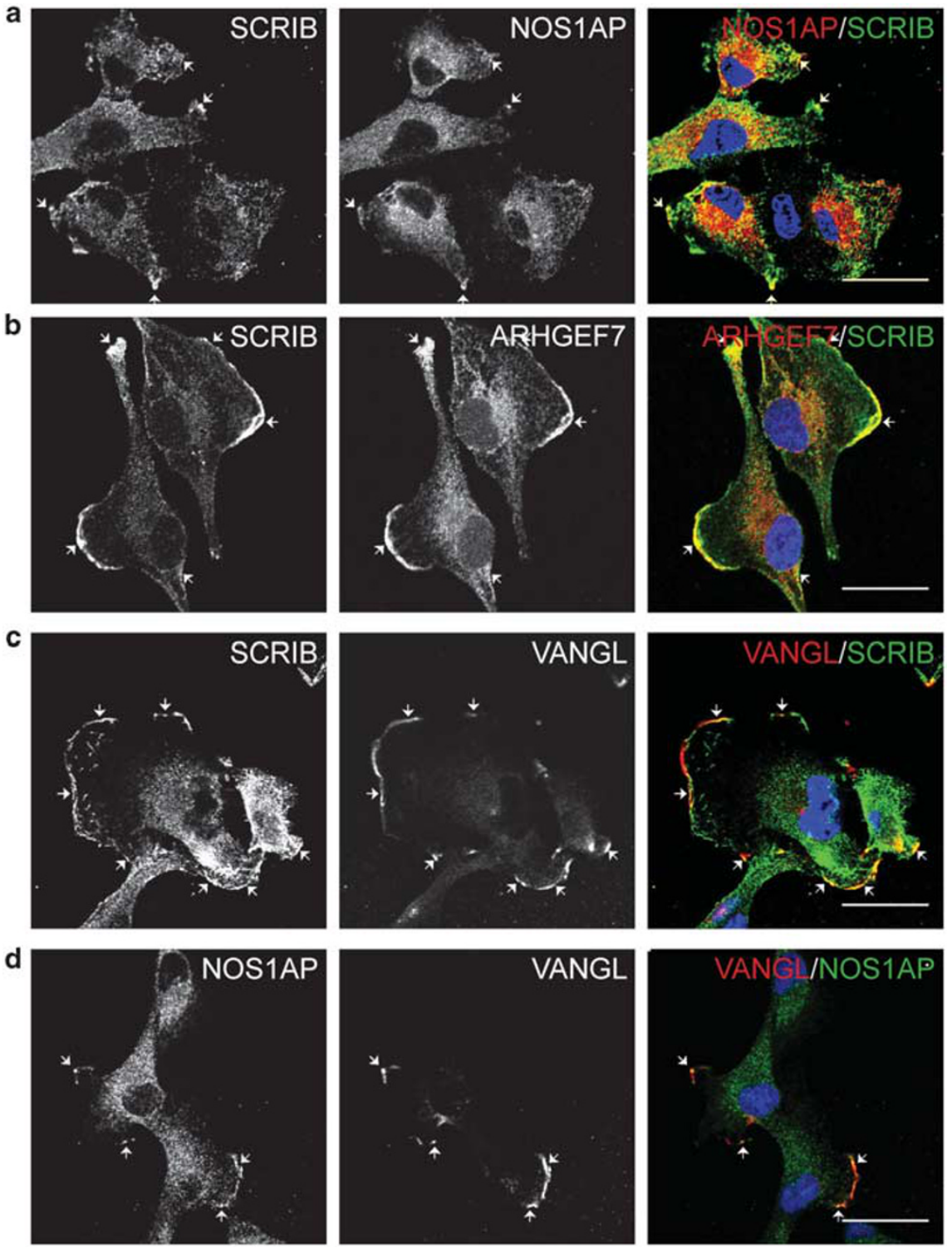

Figure 4 Subcellular localization of the SCRIB protein interaction network. (a-d) Confocal images of MDA-MB-231 cells stained with the following antibodies to detect endogenous SCRIB-associated proteins: (a) SCRIB and NOS1AP, (b) SCRIB and ARHGEF7, (c) SCRIB and VANGL, or (d) NOS1AP and VANGL. Signals from individual antibodies are shown in the left and center columns, whereas a pseudo-colored merged image is included in the right column, with 4,6-diamidino-2-phenylindole (DAPI) DNA stain shown in blue. Areas of colocalization (yellow color) are indicated by white arrows. In all images scale bars are $30 \mu \mathrm{m}$.

that most of the NOS1AP protein co-fractionates with SCRIB in the concanavalin-A flow-through, which contains non-plasma membrane proteins from both MCF10a and MDA-MB-231 cells (Figures 5d and e).

SCRIB, NOSIAP and VANGL1 regulate the migration of human breast cancer cells

In light of the roles for SCRIB in the polarized migration of a variety of cell types (Qin et al., 2005; Osmani et al., 2006; Dow et al., 2007; Nola et al., 2008; Phua et al., 2009), and given the evidence above that NOS1AP is detected in complexes with SCRIB in some contexts, we next asked whether decreasing NOS1AP protein levels mimics the effects of decreasing
SCRIB in altering the motility of breast cancer cells. We reduced NOS1AP by infecting MDA-MB-231 and MCF-7 cells with two independent shRNAs, and verified knockdown by immunoblotting and by quantitative reverse transcription-PCR (Figure 6a, and Supplementary Figures S4a and b). We then assessed the migratory capacity of SCRIB and NOS1AP knockdown cell lines using two different methods. First, we monitored the migration of individual cells in a transwell assay and found that both SCRIB and NOS1AP shRNAs reduced the ability of cells to migrate in response to a chemoattractant cue (Figure 6b). Transiently transfecting MDA-MB-231 cells with a small interfering RNA targeting NOS1AP similarly reduced migration in a transwell assay, suggesting that 

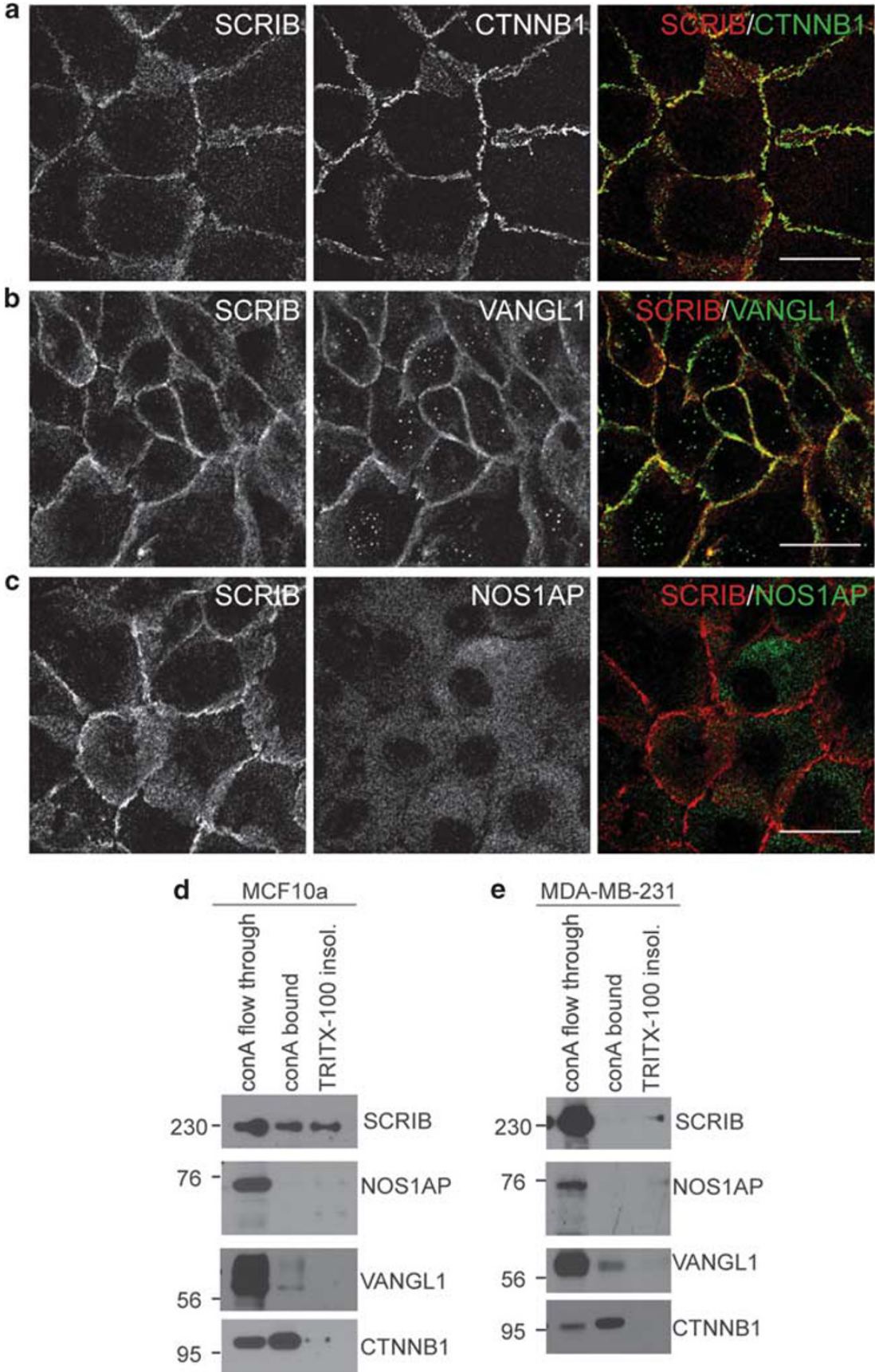

Figure 5 NOS1AP is undetectable at cell junctions in mammary cells. (a-c) Confocal microscopy of confluent MCF10a cell monolayers stained with the following antibodies: (a) SCRIB and CTNNB1, (b) SCRIB and VANGL1, and (c) SCRIB and NOS1AP. Signals from individual antibodies are shown in the left and center columns, and a pseudo-colored merged image is included in the right column. Scale bars are $30 \mu \mathrm{m}$. (d-e) Concanavalin-A fractionation of (d) MCF10a and (e) MDA-MB-231 cells lysed with Triton-X 100 buffer to identify (1) proteins associated with the plasma membrane (lane-2); (2) proteins that were not associated with the plasma membrane under these conditions (lane-1); and (3) proteins insoluble in this lysis buffer, which were re-suspended in $6 \mathrm{~m}$ urea (lane-3).

this change in cell behavior was not due to nonspecific effects of long-term shRNA expression (Supplementary Figures S4c and d). Second, we investigated the ability of stable shRNA cell lines to migrate in a wound-healing migration assay, and observed a consistent reduction in wound closure using SCRIB and NOS1AP shRNAs in both MDA-MB-231 and MCF-7 cell lines (Figures 6c-e). As SCRIB, NOS1AP and
VANGL1 associate in a protein complex, we wondered whether VANGL1 might also regulate breast cancer migration. We therefore generated additional MDAMB-231 cell lines using shRNAs targeting VANGL1 and verified knockdown by reverse transcription-PCR (Figure 6f). Like SCRIB and NOS1AP, knockdown of VANGL1 impaired MDA-MB-231 migration in a transwell assay (Figure 6g). 

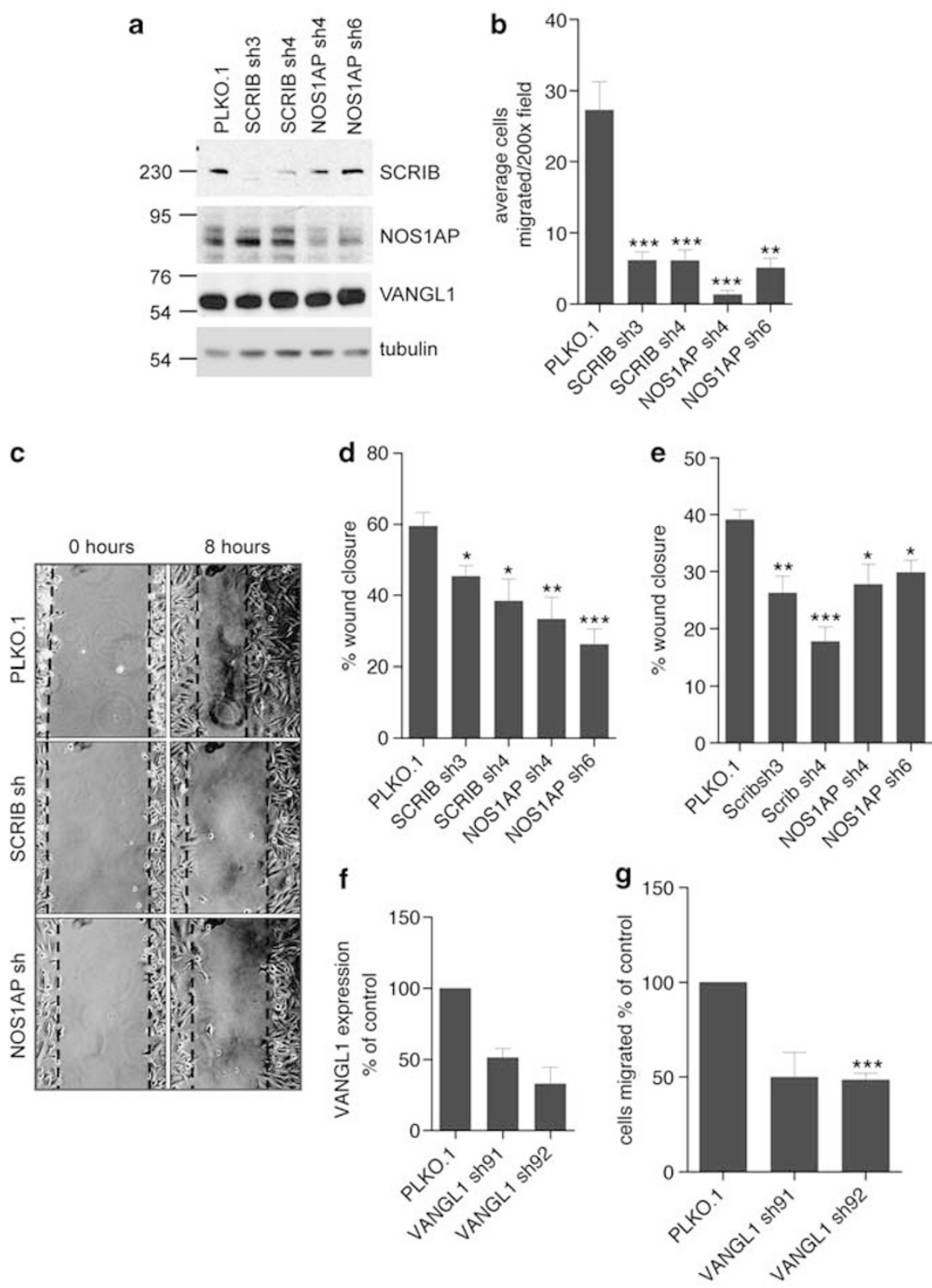

Figure 6 SCRIB, NOS1AP and VANGL1 regulate the migration of human breast cancer cells. (a) Western blot of lysates from MDAMB-231 cells infected with various shRNAs probed with antibodies for SCRIB, NOS1AP, VANGL1 and $\beta$-tubulin. (b) Summary of transwell migration of MDA-MB-231 shRNA cell lines from low-serum media to EGF media (20 ng/ml) for a period of $6 \mathrm{~h}$. The data represent average number of cells migrated per $\times 200$ microscopic field \pm s.e.m. from four independent experiments. ${ }^{*} P<0.05,{ }^{*} P<0.01$ and $* * * P<0.001$ (Student's $t$-test). (c) Representative images of MDA-MB-231 monolayers immediately and $8 \mathrm{~h}$ after wounding with a pipette tip. (d) Summary of MDA-MB-231 wound-healing migration data from three independent experiments with duplicate scratches for each shRNA cell line. The data represent average wound closure \pm s.e.m. $* P<0.05,{ }^{* *} P<0.01$ and $* * * P<0.001$ (Student's $t$-test). (e) Summary of MCF-7 breast cancer cell wound-healing migration data from three independent experiments with duplicate scratches for each shRNA cell line. The data represent average wound closure \pm s.e.m. $* P<0.05, * * P<0.01$ and $* * * P<0.001$ (Student's $t$-test). (f) Relative VANGL1 expression determined by quantitative reverse transcription-PCR of cDNA from MDA-MB-231 cells expressing control or VANGL1 shRNAs. (g) Summary of transwell migration of MDA-MB-231 cells expressing either control or VANGL1 shRNAs to medium containing $10 \%$ bovine serum for a period of $24 \mathrm{~h}$. $* * * P<0.001$ (Student's $t$-test).

SCRIB and NOSIAP regulate cell polarity during woundhealing migration

Having observed defects in the rate of cell migration with both SCRIB and NOS1AP shRNAs, we next evaluated whether reducing SCRIB and NOS1AP can impair the proper orientation of migratory cells. First, we assessed the establishment of leading-trailing polar- ity in MDA-MB-231 cells by scoring Golgi re-orientation in wound-healing assays. Four hours after wounding, control shRNA-infected cells showed an increase in the fraction of properly oriented Golgi from $\sim 30$ to $\sim 65 \%$, and developed lamellipodia demarcated by actin stress fibers running parallel to the wound edge (Figures 7c and d). Quantification of Golgi re-orientation 

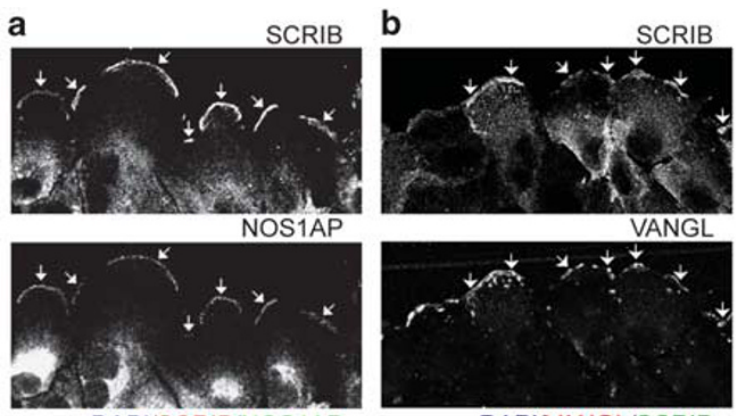

C
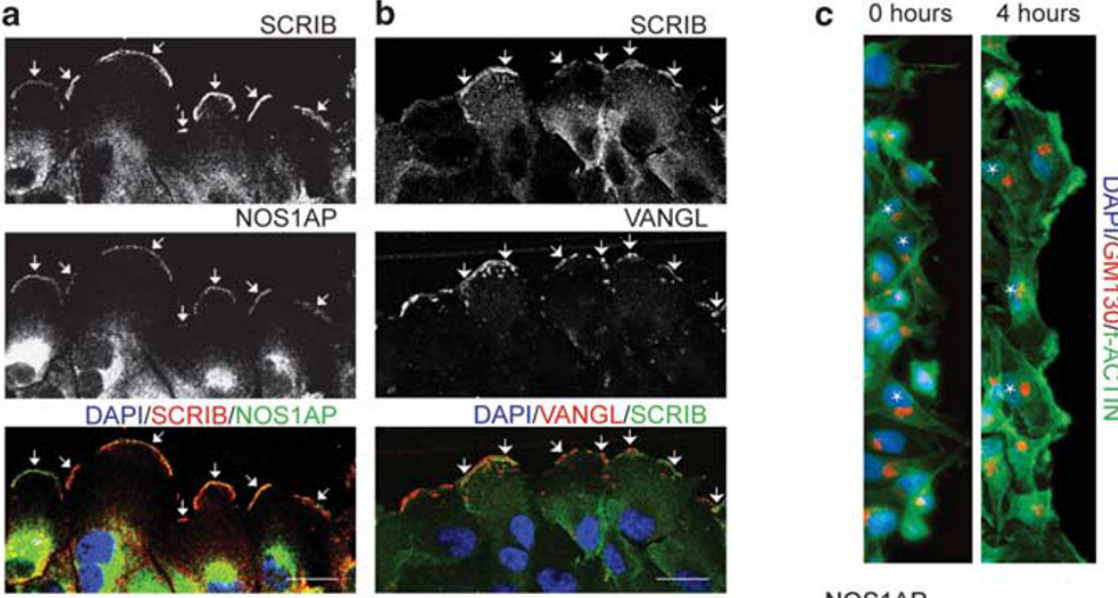

d

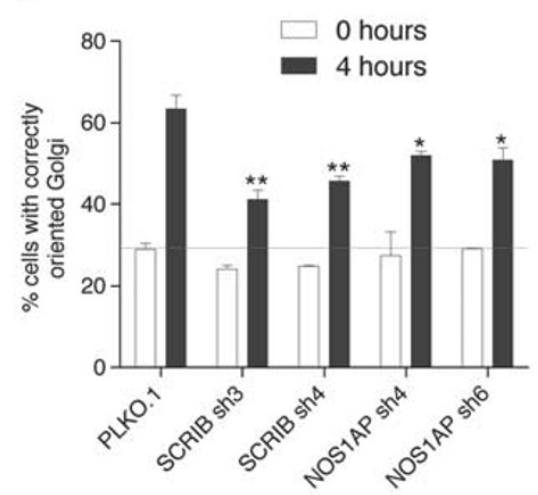

e

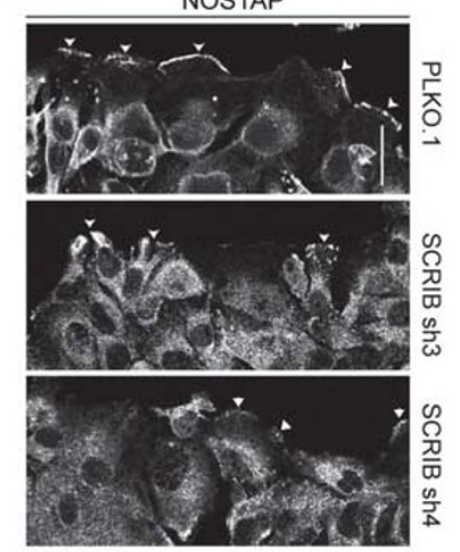

f
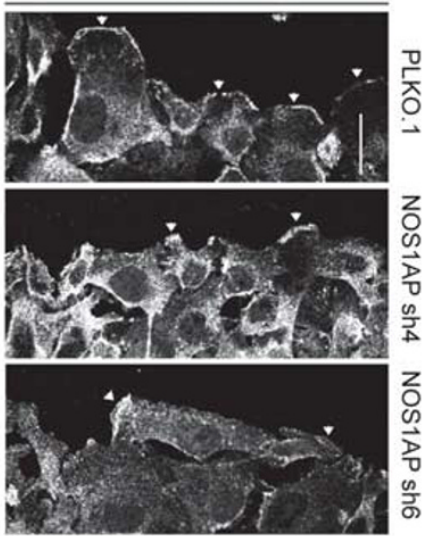

g VANGL1

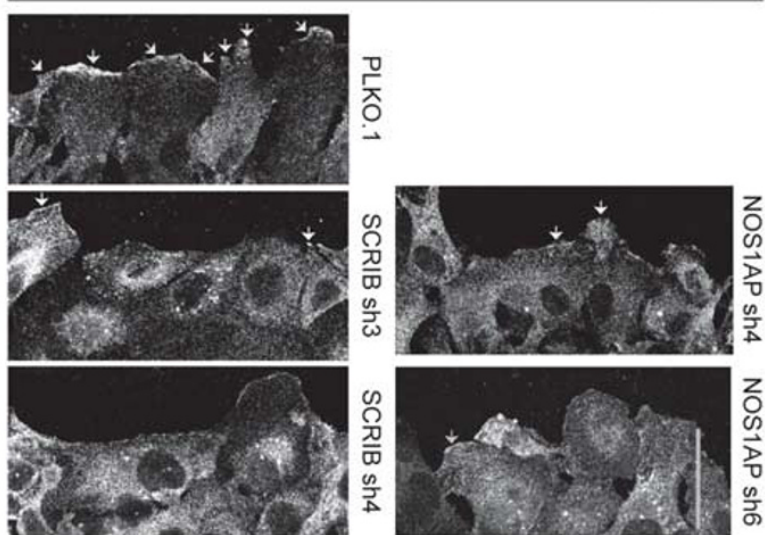

Figure 7 SCRIB and NOS1AP regulate cell polarity during wound-healing migration. (a, b) Representative images of MDA-MB-231 monolayers, which were fixed $4 \mathrm{~h}$ after wounding to induce cell migration. Fixed cells were incubated with antibodies detecting (a) SCRIB and NOS1AP, and (b) SCRIB and VANGL. Images are oriented to show cell lamellipodia extending toward the wound edge, which runs parallel to the top of each image. Arrows indicate areas where SCRIB and NOS1AP, or SCRIB and VANGL, colocalize to the leading edge. (c) MDA-MB-231 cells were fixed immediately or $4 \mathrm{~h}$ after wounding and then incubated with a GM130 antibody to visualize the Golgi apparatus, and phalloidin to visualize the actin cytoskeleton. Cells with correctly oriented Golgi relative to the nucleus and the wound edge are marked by a white asterisk. (d) Percent of MDA-MB-231 shRNA-expressing cells with proper Golgi orientation determined immediately and $4 \mathrm{~h}$ after wounding. The horizontal line indicates the level of Golgi polarization at the beginning of wound-healing migration. The data represent the mean of four independent experiments \pm s.e.m. ${ }^{*} P<0.05$ and ${ }^{* *} P<0.01$ (Student's $t$-test). See Materials and methods for more information on Golgi polarity scoring. (e) Confocal images of wounded MDA-MB-231 cell monolayers expressing either PLKO.1 (top panel) or SCRIB shRNAs stained with an NOS1AP antibody. (f) Confocal images of wounded MDA-MB-231 cell monolayers expressing either PLKO.1 (top panel) or NOS1AP shRNAs stained with a SCRIB antibody. (g) Confocal images of wounded MDA-MB-231 cell monolayers expressing PLKO.1, SCRIB or NOS1AP shRNAs stained with a VANGL antibody. In panels $\mathbf{e}-\mathbf{g}$ arrows indicate areas where SCRIB, NOS1AP or VANGL localize to the leading edge. In all images scale bars are $30 \mu \mathrm{m}$. 
revealed that SCRIB and NOS1AP shRNAs significantly reduced the establishment of organelle polarity during wound-healing migration (Figure $7 \mathrm{~d}$ ).

To better understand the roles of SCRIB and NOS1AP in leading-trailing polarity, we used confocal microscopy to detect SCRIB-associated proteins in MDA-MB-231 cell monolayers after wounding to induce cell migration. We found that SCRIB, NOS1AP and VANGL asymmetrically localize to the leading edge of cell lamellipodia extending into the wound (Figures $7 \mathrm{a}$ and $\mathrm{b}$ ). However, when we analyzed the localization of SCRIB and NOS1AP in the knockdown cells, we found that SCRIB shRNAs disrupt the localization of NOS1AP to the leading edge of lamellipodia, resulting in a more uniform distribution of NOS1AP throughout the cytoplasm (Figure 7e). NOS1AP shRNAs also disrupted the sub-cellular distribution of SCRIB by similarly preventing the localization of SCRIB to the leading edge of lamellipodia (Figure 7f). As SCRIB, NOS1AP and VANGL associate in a protein complex, we wondered whether the levels of SCRIB and NOS1AP might influence the subcellular localization of VANGL1. Compared with control, SCRIB and NOS1AP shRNAs also partially reduced VANGL1 localization to the leading edge (Figure $7 \mathrm{~g}$ ).

\section{NOSIAP protein is reduced in breast cancer metastases from lymph nodes}

We next asked if NOS1AP regulates other cellular behaviors related to breast cancer progression. First, we assessed the proliferation of cells expressing SCRIB and NOS1AP shRNAs in two-dimensional culture and found no significant difference in either MCF-7 or MDA-MB-231 cells (Figures 8a and b). Next, we assessed the anchorage-independent growth of MDA-MB-231 shRNA cell lines. In contrast to twodimensional culture, we found that NOS1AP shRNAexpressing cells formed approximately twice as many colonies in soft agar compared with PLKO.1 controls, whereas SCRIB shRNAs did not significantly affect growth in this assay (Figures $8 \mathrm{c}$ and $\mathrm{d}$ ).

As in vitro and mouse models of human cancers cannot fully recapitulate the complexities of human disease, we next analyzed human breast cancer specimens to determine whether NOS1AP and SCRIB protein expression or localization patterns were altered. This study included a variety of different breast tumors: patient samples with and without ductal carcinoma in situ, and patient samples with and without lymph node metastases (Supplementary Table S5). As others have observed (Navarro et al., 2005; Zhan et al., 2008), SCRIB was present in normal tissues and was highly expressed in invasive tumors with a combination of membranous and cytosolic localization (Figure 8e and Supplementary Table S6). SCRIB staining intensity did not correlate with ER/PR/HER2 status, the presence of metastatic lesions or the grade of breast cancer samples, and was also strongly expressed in primary metastases taken from the lymph nodes (Supplementary Tables S6 and S8).
In order to investigate the relationship between NOS1AP expression and clinical outcomes in breast cancer, we analyzed several multiple tumor microarray data sets from breast cancer patients. Unlike $S C R I B$ and $V A N G L 1$ expression (Figure 1), there were no statistically significant associations between NOSIAP transcript levels and clinical outcomes (Supplementary Figure S5). Next, we took additional sections of patient samples and performed immunohistochemistry to determine the level and localization of NOS1AP protein during cancer progression. In both normal and tumor tissue, NOS1AP was localized to the cytoplasm and was expressed at approximately the same level regardless of the breast cancer subtype, the ER/PR/HER2 status or the presence of lymph node metastases (Figure $8 \mathrm{e}$ and Supplementary Table S7). Significantly, when we analyzed primary metastatic lesions, we found that levels of NOS1AP were reduced or undetectable in half of the cases analyzed (Figure 8e and Supplementary Table S8). This represents the first evidence that NOS1AP levels are altered in human breast cancer.

\section{Discussion}

Several studies that have analyzed SCRIB expression in tumors have led to somewhat conflicting results. Navarro et al. (2005) reported that SCRIB levels were reduced in lobular breast carcinomas, whereas levels were neither elevated nor consistently reduced in ductal carcinomas. In an independent study, Zhan et al. (2008) found no difference in SCRIB protein levels in breast cancer samples, but found that small interfering RNAmediated reduction of SCRIB promotes the growth of mouse mammary tumors in vivo. In the present study, we observed that reducing the levels of SCRIB slows the growth of human breast cancer cells in mouse xenografts, and found that high expression of SCRIB in breast cancer patient samples correlates with relapse and decreased survival. Together, these data suggest that breast cancer progression may be sensitive to the levels of SCRIB.

We hypothesized that the ability of SCRIB to influence breast cancer progression might involve changes in the proteins with which SCRIB associates. We therefore characterized SCRIB protein complexes by mass spectrometry and found that SCRIB exists in multiple pools within cells, associating with a complex of SCRIB-ARHGEF-PAK-GIT proteins and, also, with a complex of SCRIB-NOS1AP-VANGL proteins.

In invasive breast cancer cells we find that SCRIB, NOS1AP and VANGL1 colocalize along the edge of cellular protrusions. These data support the mass spectrometry data by showing that proteins, which associate with one another in cell lysates, are normally found in overlapping domains within cells. By contrast, we find that SCRIB and VANGL1 localize to sites of cell-cell contact in normal mammary cells, whereas NOS1AP remains in the cytosol. The localization of NOS1AP at cell protrusions in invasive breast 

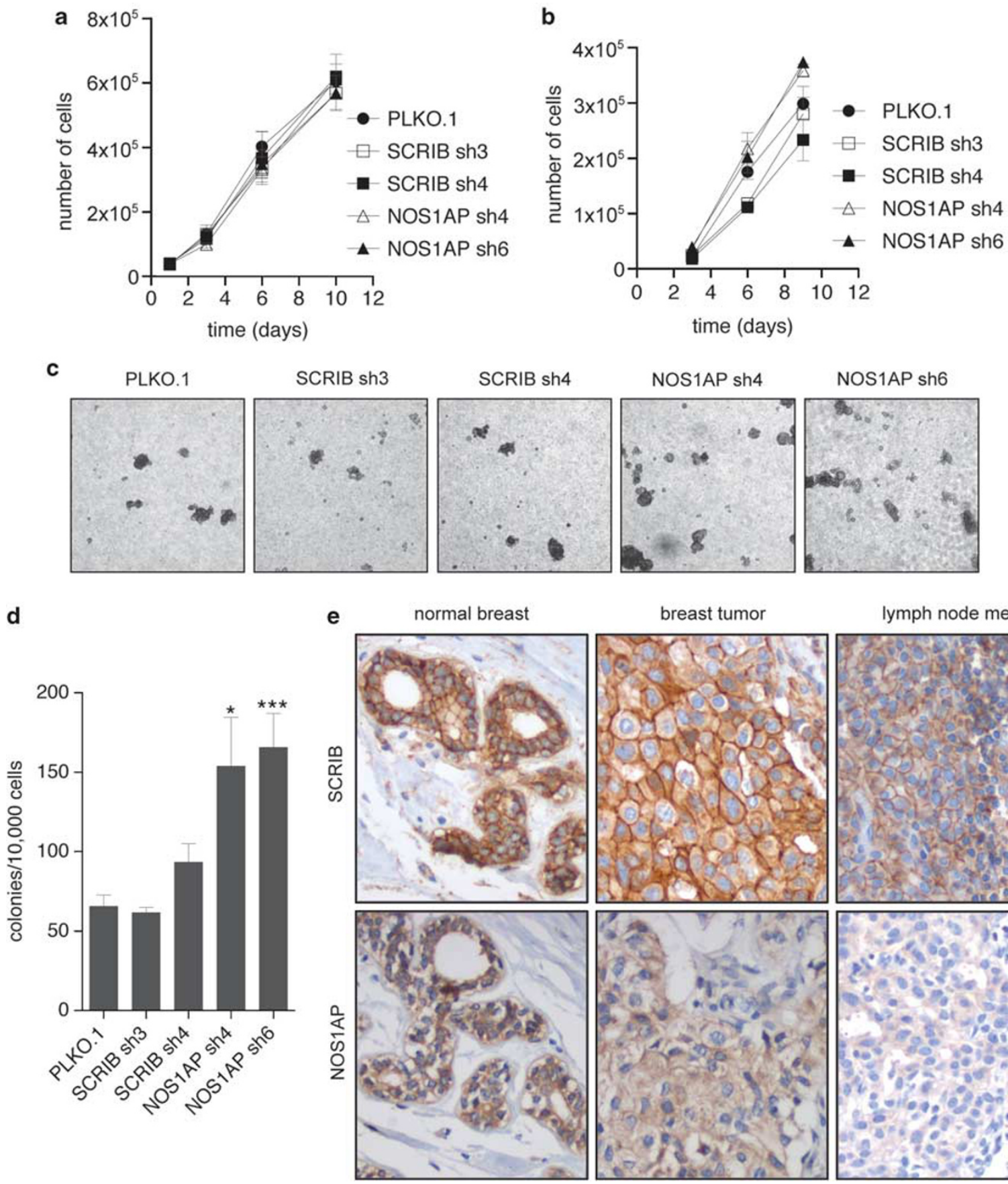

lymph node met.

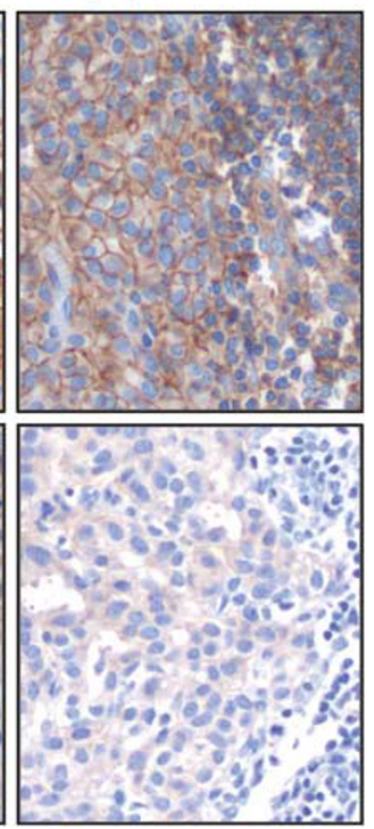

Figure 8 NOS1AP protein is reduced in breast cancer metastases from lymph nodes. (a) Growth curves of MDA-MB-231 shRNA cell lines grown in two dimensions $(n=3)$. (b) Growth curves of MCF-7 shRNA cell lines grown in two dimensions $(n=3)$. (c) Representative low-power microscopic images of MDA-MB-231 cells infected with various shRNAs growing as colonies in $0.3 \%$ agar for anchorage-independent growth assay. (d) Quantification of colonies formed per 10000 MDA-MB- 231 cells plated in $0.3 \%$ agar after 3 weeks. The data represent the mean of three independent experiments performed in triplicate. ${ }^{*} P<0.05$ and $* * * P<0.001$ (Student's $t$-test). (e) Representative images of normal mammary tissue (left panels), breast cancer (middle panels) and lymph node metastatic lesions (right panels) stained for SCRIB and NOS1AP. Cell nuclei were detected by counterstaining with hematoxylin.

cancer cells led us to hypothesize that NOS1AP might cooperate with SCRIB and VANGL1 to promote cell migration. Indeed, our results revealed a functional relevance for SCRIB-NOS1AP-VANGL1 protein complexes in breast cancer cells because loss of SCRIB, NOS1AP or VANGL1 slows migration. Several studies now suggest that SCRIB may promote cell migration by acting as a scaffold for recruitment of ARHGEFs and PAKs to the leading edge (Osmani et al., 2006; Dow et al., 2007), and by acting as a determinant of organelle polarity (Osmani et al., 2006; Dow et al., 2007). As NOS1AP analogously acts as a signaling adapter 
protein to localize active nNOS and RAC1 to the plasma membrane in neurons (Jaffrey et al., 1998; Fang et al., 2000; Jaffrey, 2002; Carrel et al., 2009; Richier et al., 2010), we then asked whether NOS1 AP might also have a role in the establishment of leading-trailing polarity. We found that SCRIB and NOS1AP are mutually dependent in terms of their localization to the leading edge of breast cancer cells. Furthermore, knockdown of either SCRIB or NOS1AP prevents the localization of VANGL at the leading edge as well as the re-orientation of the Golgi apparatus. Alternatively, it is also possible that SCRIB and NOS1AP shRNAs indirectly result in defective leading-trailing polarity of SCRIB, NOS1AP, and VANGL owing to a generalized loss of cell polarity. Collectively, these data indicate that tightly controlled levels of SCRIB, NOS1AP and VANGL proteins are necessary to regulate cell polarity and motility.

Recent work in Drosophila demonstrates that expression of tumor necrosis factor and constitutively activated RASV12 can convert SCRIB from a tumor suppressor to enhancer of tissue overgrowth (Cordero et al., 2010), which suggests that SCRIB protein complexes may regulate cancer progression in a context-dependent manner. This study finds that other members of SCRIB protein complexes may also act in a context-dependent manner. Although we find that reducing NOS1AP slows cell migration as is typical of a metastasis suppressor, we paradoxically observe that NOS1APdepleted cells outperform control cell lines in anchorageindependent growth assays. It is possible that NOS1AP may have a unique function in promoting three-dimensional growth that is independent of SCRIB, as SCRIB shRNAs did not modulate soft agar colony formation.

We also analyzed the expression of SCRIB and SCRIB-associated proteins in samples from a variety of breast cancer lesions, and note a reduction in NOS1AP in lymph node metastases compared with primary tumors and normal breast. It is unclear whether NOS1AP is lost as cells leave the primary tumor and invade the lymphatic system, or if loss of NOS1AP occurs as a result of different environmental conditions in the lymph node. Nevertheless, our results suggest that SCRIB- and NOS1AP-associated protein complexes may be involved in the progression of breast cancer.

\section{Materials and methods}

Cell culture and generation of stable cell lines

Cell lines were purchased from ATCC and cultured according to the vendor's instructions. shRNAs in a PLKO.1 backbone were purchased from Open Biosystems (Huntsville, AL, USA). Polyclonal shRNA cell lines were generated by infecting cells with lentivirus and selecting with $2 \mu \mathrm{g} / \mathrm{ml}$ puromycin. NOS1AP, ARHGEF7 and SCRIB were PCR-amplified from cDNA and cloned into pcDNA3.1 at the $3^{\prime}$ end of a FLAG tag for transient transfection using Lipofectamine LTX (Invitrogen, Carlsbad, CA, USA), or at the $3^{\prime}$ end of a GFP tag in a pPHAGE vector backbone for lentivirus production. GFP-NOS1AP- and GFP-expressing cell lines were obtained by fluorescence-activated cell sorting after infection.
Mass spectrometry and western blotting

TAP-TAG SCRIB and associated proteins were isolated from HEK293 T cells as described previously (Angers et al., 2006; Major et al., 2007). For endogenous SCRIB pull downs, cleared protein extracts generated from $1 \times 10^{9}$ HEK293 $\mathrm{T}$ cells were incubated with $10 \mu \mathrm{g}$ of SCRIB antibody bound to protein-G-sepharose at $4{ }^{\circ} \mathrm{C}$ for $2 \mathrm{~h}$. After extensive washing, antibody complexes were eluted with sodium citrate buffer (pH 2.0) and analyzed by tandem mass spectrometry as described previously (Angers et al., 2006).

\section{Co-immunoprecipitation}

A total of $1.0 \times 10^{6}$ MDA-MB-231 cells were lysed in buffer containing $50 \mathrm{~mm}$ Tris (8.0), $150 \mathrm{~mm} \mathrm{NaCl}, 0.1 \%$ Na-deoxycholate, $1 \%$ NP-40, and protease and phosphatase inhibitors. Cleared lysates were combined with $0.5 \mu \mathrm{g}$ of one of GFP, ARHGEF7, FLAG, SCRIB or IgG control antibodies, and incubated at $4{ }^{\circ} \mathrm{C}$ for $2 \mathrm{~h}$. Immune complexes were precipitated with protein-Gsepharose, washed extensively and then eluted with $1 \times$ NUPAGE loading buffer for analysis by western blotting.

\section{Concanavalin- $A$ fractionation}

MDA-MB-231 or MCF10a cells were lysed in a buffer containing 1\% Triton-X 100, $20 \mathrm{~mm}$ Tris- $\mathrm{HCl}$ (7.5), $150 \mathrm{~mm}$ $\mathrm{NaCl}$, and protease and phosphatase inhibitors. Proteins were solubilized on a rotator at $4{ }^{\circ} \mathrm{C}$ for $1 \mathrm{~h}$ before clearing by centrifugation. The soluble fraction was incubated with concanavalin-A beads on a rotator at $4{ }^{\circ} \mathrm{C}$ for $2 \mathrm{~h}$ at which point flow-through fractions were collected. After washing with lysis buffer, concanavalin-A-bound proteins were eluted with $1 \times$ NUPAGE loading buffer for western blotting.

\section{Immunofluorescence}

Cells plated on fetal bovine serum-coated coverslips were fixed with $4 \%$ paraformaldehyde diluted in phosphate-buffered saline ( $\mathrm{pH} 7.0)$ for $10 \mathrm{~min}$ and blocked with $4 \%$ bovine serum albumin in phosphate-buffered saline $+0.1 \%$ Tween- $20(\mathrm{pH}$ 7.0) before incubation with one, or more, of the following primary antibodies: SCRIB (Santa Cruz Biotechnology, Santa Cruz, CA, USA, H300), NOS1AP (Santa Cruz Biotechnology, R300), VANGL1 (Sigma-Aldrich, St Louis, MO, USA), VANGL (in-house), ARHGEF7 (Chemicon, Temecula, CA, USA) or GM130 (Stressgen/Enzo Life Sciences, Exeter, UK). Secondary fluorophore-conjugated antibodies were used in combination with fluorophore-conjugated phalloidin (Invitrogen). Cells were imaged by confocal microscopy with a Nikon Eclipse imaging system using a $\times 60$ oil-immersion objective.

\section{Cell migration assays}

For transwell assays, 50000 cells were plated in the upper chamber of a transwell $(8 \mu \mathrm{m}$ pore $)$ with $20 \mathrm{ng} / \mathrm{ml}$ epidermal growth factor (EGF) or $10 \%$ fetal bovine serum provided in the lower chamber. Cells remaining in the upper chamber were removed and cells that had migrated to the bottom of the membrane were fixed, stained with crystal violet and counted. For wound-healing assays, confluent MDA-MB-231 or MCF7 cells were wounded with a pipette tip and imaged at various time points with a Nikon Eclipse TS100 microscope. Wounds were measured at six or more locations in order to determine the width of each replicate wound.

Golgi orientation assays

Wounded MDA-MB-231 monolayers were stained with GM130 antibodies and phalloidin and imaged with a Nikon Eclipse wide-field microscope. Images from four independent 
experiments were analyzed in a blinded manner to determine Golgi polarity essentially as described by Nobes (1999). Briefly, cells immediately adjacent to the wound were scored as polarized when the Golgi resided in the 120 degree sector between the nucleus and the leading edge, and scored as unpolarized if the Golgi was either diffusely distributed or was not localized to the 120 degree sector between the nucleus and the leading edge. At least 150 cells were scored per shRNA per experiment in order to determine the percent of cells with polarized Golgi, which was calculated by dividing the number of polarized cells by the total cells scored for each shRNA.

\section{Cell proliferation experiments}

For assays of two-dimensional growth, 30000 MDA-MB-231 or MCF-7 cells were seeded in each well of a 12-well plate and fed with a change of media every 3 days. Cells were later collected in phosphate-buffered saline/EDTA after 1, 3, 6 or 9 days, and counted. For anchorage-independent growth assays, 10000 MDA-MB-231 cells in $0.3 \%$ agarose-containing growth media were plated over a base $0.6 \%$ base agarose. After 3 weeks colonies with $>25$ cells were scored.

\section{Xenografts}

MDA-MB-231 cells were re-suspended at a concentration of $1 \times 10^{7} \mathrm{cells} / \mathrm{ml}$ and $0.1 \mathrm{ml}$ of cells were injected into the mammary fat pads of NOG or athymic nude immunocompromised mice (Harlan Labs, Indianapolis, IN, USA). The growth of primary tumors was monitored biweekly using the following formula: Volume $=(\pi / 6) \times a^{2} b$, where $a$ is the short diameter and $b$ is the long diameter.

\section{Immunohistochemistry of breast cancer samples}

Breast cancer patient samples were obtained from the University of Washington, Department of Pathology. Tumor sections were deparaffinized followed by antigen retrieval by microwaving slides in EDTA buffer $(\mathrm{pH}$ 8.0). Samples were then incubated with NOS1AP (1:60 dilution; Santa Cruz Biotechnology, R300) or SCRIB antibodies (1:100; Santa Cruz Biotechnology, C20) for $40 \mathrm{~min}$, followed by incubation with a biotinylated $\mathrm{mIgG}$ secondary antibody for $30 \mathrm{~min}$ and detection using an ABC kit (avidin biotin complex with peroxidase) for $30 \mathrm{~min}$. Color was developed with diaminobenzidine and hematoxylin before dehydration through graded alcohols and mounting. Tumor grade was scored using a Nottingham system and the ER/PR status of tumors was determined using the Allred scale as described previously (Harvey et al., 1999). The intensity $(0-3+)$ and percent of SCRIB and NOS1AP positive cells were determined by a blinded board certified pathologist (KHA). All human studies were approved by an institutional review board and the Department of Health and Human Services.

\section{Statistical analysis}

All migration and growth assays were repeated at least three times in duplicate and significance was assessed using unpaired Student's $t$-test. Error bars show s.e.m. ( ${ }^{*} P<0.05$, ${ }^{* *} P<0.01$ and $* * * P<0.001)$. For Kaplan-Meier survival analysis, significance of the association between transcript expression and disease outcome was determined by using the GraphPad statistical software and Mantel-Cox log-rank test.

\section{Generation of VANGL antibodies}

Peptide VANGL2 antibodies were generated by Aves Labs Inc. (Tigard, OR, USA) (peptide sequence: CZ RRR DNS HNE YYY EEA EHE RR), and validated by ELISA and western blotting (data not shown). As this peptide is $>90 \%$ identical to the amino-acid sequence of VANGL1, we, therefore, refer to this antibody as VANGL throughout the text (Supplementary Figure S1).

\section{Conflict of interest}

The authors declare no conflict of interest.

\section{Acknowledgements}

Funding for this research was provided by the US Department of Defense Breast Cancer Research Award (Grant W81XWH070100367). RTM is an investigator of the Howard Hughes Medical Institute. MR holds a postdoctoral fellowship from the Canadian Breast Cancer Foundation-Ontario Region. Special thanks to Allison Adams for providing the GFP viruses, and Ron Steifert and the Lynn and Mike Garvey Imaging Center for their assistance.

\section{References}

Angers S, Thorpe CJ, Biechele TL, Goldenberg SJ, Zheng N, MacCoss MJ et al. (2006). The KLHL12-Cullin-3 ubiquitin ligase negatively regulates the Wnt-beta-catenin pathway by targeting Dishevelled for degradation. Nat Cell Biol 8: 348-357.

Audebert S, Navarro C, Nourry C, Chasserot-Golaz S, Lécine P, Bellaiche Y et al. (2004). Mammalian Scribble forms a tight complex with the betaPIX exchange factor. Curr Biol 14: 987-995.

Bild AH, Yao G, Chang JT, Wang Q, Potti A, Chasse D et al. (2006). Oncogenic pathway signatures in human cancers as a guide to targeted therapies. Nature 439: 353-357.

Bilder D, Li M, Perrimon N. (2000). Cooperative regulation of cell polarity and growth by Drosophila tumor suppressors. Science 289: 113-116.

Bilder D, Perrimon N. (2000). Localization of apical epithelial determinants by the basolateral PDZ protein Scribble. Nature 403: 676-680. 
during directed epithelial migration: regulation of Rho GTPase recruitment to the leading edge. Oncogene 26: 2272-2282.

Fang M, Jaffrey SR, Sawa A, Ye K, Luo X, Snyder SH. (2000). Dexras1: a $G$ protein specifically coupled to neuronal nitric oxide synthase via CAPON. Neuron 28: 183-193.

Harvey JM, Clark GM, Osborne CK, Allred DC. (1999). Estrogen receptor status by immunohistochemistry is superior to the ligandbinding assay for predicting response to adjuvant endocrine therapy in breast cancer. J Clin Oncol 17: 1474-1481.

Ivanov AI, Young C, Den Beste K, Capaldo CT, Humbert PO, Brennwald $\mathrm{P}$ et al. (2010). Tumor suppressor scribble regulates assembly of tight junctions in the intestinal epithelium. Am J Pathol 176: $134-145$.

Jaffrey SR. (2002). Neuronal nitric-oxide synthase localization mediated by a ternary complex with synapsin and CAPON. Proc Natl Acad Sci USA 99: 3199-3204.

Jaffrey SR, Snowman AM, Eliasson MJ, Cohen NA, Snyder SH. (1998). CAPON: a protein associated with neuronal nitric oxide synthase that regulates its interactions with PSD95. Neuron 20: $115-124$

Major MB, Camp ND, Berndt JD, Yi X, Goldenberg SJ, Hubbert C et al. (2007). Wilms tumor suppressor WTX negatively regulates WNT/beta-catenin signaling. Science 316: 1043-1046.

Métais J-Y, Navarro C, Santoni M-J, Audebert S, Borg JP. (2005). hScrib interacts with ZO-2 at the cell-cell junctions of epithelial cells. FEBS Lett 579: 3725-3730.

Montcouquiol M, Rachel RA, Lanford PJ, Copeland NG, Jenkins NA, Kelley MW. (2003). Identification of Vangl2 and Scrb1 as planar polarity genes in mammals. Nature 423: 173-177.

Moreau MM, Piguel N, Papouin T, Koehl M, Durand CM, Rubio ME et al. (2010). The planar polarity protein Scribblel is essential for neuronal plasticity and brain function. J Neurosci $\mathbf{3 0}$ : 9738-9752.

Murdoch JN, Henderson DJ, Doudney K, Gaston-Massuet C, Phillips HM, Paternotte C et al. (2003). Disruption of scribble (Scrb1) causes severe neural tube defects in the circletail mouse. Hum Mol Genet 12: 87-98.

Murdoch JN, Rachel RA, Shah S, Beermann F, Stanier P, Mason CA et al. (2001). Circletail, a new mouse mutant with severe neural tube defects: chromosomal localization and interaction with the loop-tail mutation. Genomics 78: 55-63.

Nobes CD. (1999). Rho GTPases control polarity, protrusion, and adhesion during cell movement. $J$ Cell Biol 144: $1235-1244$.

Nola S, Sebbagh M, Marchetto S, Osmani N, Nourry C, Audebert S et al. (2008). Scrib regulates PAK activity during the cell migration process. Hum Mol Genet 17: 3552-3565.
Navarro C, Nola S, Audebert S, Santoni M-J, Arsanto J-P, Ginestier $\mathrm{C}$ et al. (2005). Junctional recruitment of mammalian Scribble relies on E-cadherin engagement. Oncogene 24: 4330-4339.

Osmani N, Vitale N, Borg J-P, Etienne-Manneville S. (2006). Scrib controls Cdc42 localization and activity to promote cell polarization during astrocyte migration. Curr Biol 16: 2395-2405.

Petit MMR, Crombez KRMO, Vervenne HBVK, Weyns N, Van de Ven WJM. (2005a). The tumor suppressor Scrib selectively interacts with specific members of the zyxin family of proteins. FEBS Lett 579: 5061-5068.

Petit MMR, Meulemans SMP, Alen P, Ayoubi TAY, Jansen E, Van de Ven WJM. (2005b). The tumor suppressor Scrib interacts with the zyxin-related protein LPP, which shuttles between cell adhesion sites and the nucleus. BMC Cell Biol 6: 1 .

Phua DCY, Humbert PO, Hunziker W. (2009). Vimentin regulates scribble activity by protecting it from proteasomal degradation. $\mathrm{Mol}$ Biol Cell 20: 2841-2855.

Qin Y, Capaldo C, Gumbiner BM, Macara IG. (2005). The mammalian Scribble polarity protein regulates epithelial cell adhesion and migration through $\mathrm{E}$ cadherin. $J$ Cell Biol 171: 1061-1071.

Richier L, Williton K, Clattenburg L, Colwill K, O'Brien M, Tsang C et al. (2010). NOS1AP associates with Scribble and regulates dendritic spine development. J Neurosci 30: 4796-4805.

Vervenne HBVK, Crombez KRMO, Lambaerts K, Carvalho L, Köppen M, Heisenberg CP et al. (2008). Lpp is involved in Wnt/ PCP signaling and acts together with Scrib to mediate convergence and extension movements during zebrafish gastrulation. Dev Biol 320: $267-277$.

Wada H, Iwasaki M, Sato T, Masai I, Nishiwaki Y, Tanaka H et al. (2005). Dual roles of zygotic and maternal Scribblel in neural migration and convergent extension movements in zebrafish embryos. Development 132: 2273-2285.

Wang Y, Klijn JGM, Zhang Y, Sieuwerts AM, Look MP, Yang F et al. (2005). Gene expression profiles to predict distant metastasis of lymph-node-negative primary breast cancer. Lancet 365: 671-679.

Zhan L, Rosenberg A, Bergami KC, Yu M, Xuan Z, Jaffe AB et al. (2008). Deregulation of scribble promotes mammary tumorigenesis and reveals a role for cell polarity in carcinoma. Cell 135: 865-878.

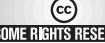
This work is licensed under the Creative Commons Attribution-NonCommercial-No Derivative Works 3.0 Unported License. To view a copy of this license, visit http://creativecommons.org/licenses/by-nc-nd/3.0/

Supplementary Information accompanies the paper on the Oncogene website (http://www.nature.com/onc) 